\title{
Rezidivierte Lymphome zielgerichtet mit Brentuximab Vedotin behandeln
}

\author{
Mit dem Antikörper-Wirkstoff- \\ Konjugat Brentuximab Vedotin \\ $\left(\right.$ Adcetris $^{\oplus}$ ) von Takeda steht seit \\ zwanzig Jahren erstmals wieder \\ eine Substanz zur Verfügung, die \\ das Potenzial hat, die Prognose \\ der Patienten zu verbessern.
}

Seit Dezember 2012 steht mit Brentuximab Vedotin eine neue Therapie für die Behandlung erwachsener Patienten mit rezidiviertem oder refraktärem $(r / r)$ CD30-positivem Hodgkin-Lymphom (HL) zur Verfügung - entweder nach autologer Stammzelltransplantation (ASCT) oder nach mindestens zwei vorangegangenen Therapien, wenn eine ASCT oder eine Kombinationschemotherapie nicht infrage kommt. Die zweite zugelassene In- dikation ist die Behandlung von erwachsenen Patienten mit r/r CD30-positivem oder systemischem anaplastischen Lymphom (sALCL).

Bei Patienten mit $\mathrm{r} / \mathrm{r}$ HL erreichte die Monotherapie mit dem neuen Medikament in der zulassungsrelevanten einarmigen multizentrischen Studie SG0350003 bei $94 \%$ der insgesamt 102 Patienten eine Reduktion der Tumorlast (75\% objektive Remissionen [OR], 33\% komplette Remissionen [CR]). Auch Patienten ohne vorherige ASCT profitieren: In entsprechenden Studien erreichten $54 \%$ dieser Patienten $(\mathrm{r} / \mathrm{r}$ HL ohne vorherige ASCT) eine objektive Tumorremission, darunter $22 \%$ CR. Knapp zwei Drittel der Patienten waren bereits im fortgeschrittenen Krankheitsstadium Grad III/IV.
Bei Patienten mit $\mathrm{r} / \mathrm{r}$ sALCL erreichte die Monotherapie mit Brentuximab Vedotin in der zulassungsrelevanten einarmigen multizentrischen Studie SG0350004 bei $97 \%$ der 58 Patienten eine Reduktion der Tumorlast. Eine objektive Remission hatten $86 \%$ der Patienten, darunter 59 \% CR. Der Effekt war unabhängig vom ALK-Status, dem Vorliegen einer B-Symptomatik oder sonstigen ungünstigen prognostischen Kriterien. In beiden Studien wurde bei etwa $60 \%$ der HL- bzw. sALCL-Patienten eine längere progressionsfreie Überlebenszeit im Vergleich zur jeweiligen unmittelbar vorangegangenen Behandlung erreicht.

Nebenwirkungen waren in der Regel klinisch zuverlässig handhabbar, meist durch Dosisreduktion. Im Vordergrund standen periphere sensorische Neuropathien bei gut $40 \%$ der Patienten, gefolgt von Übelkeit, Müdigkeit, Diarrhö und Neutropenien. Brentuximab Vedotin erwies sich in beiden Studien verglichen mit herkömmlicher Polychemotherapie als gut verträglich.

\section{Pertuzumab kann Überlebenszeit bei Brustkrebs verlängern}

\section{Pertuzumab (Perjeta ${ }^{\oplus}$ ) von Roche optimiert die gegen HER2 gerichtete Behandlung von Patientinnen mit metastasiertem HER2-positivem Mammakarzinom.}

Seit März 2013 ist Pertuzumab in Kombination mit Trastuzumab und dem Zytostatikum Docetaxel bei Patientinnen mit HER2-positivem metastasiertem oder lokal rezidivierendem, inoperablem Mammakarzinom zugelassen. Die Patientinnen dürfen für die metastasierte Erkrankung noch keine Anti-HER2-Therapie oder Chemotherapie erhalten haben, wie es auch die Einschlusskriterien der Phase-IIIZulassungsstudie CLEOPATRA (Clinical Evaluation of Pertuzumab and Trastuzumab) gefordert haben.

In dieser Studie erreichte die duale HER2-Blockade mit Pertuzumab/Trastuzumab plus Docetaxel im Vergleich mit alleiniger Trastuzumab/Docetaxel-Therapie bei insgesamt 808 Patientinnen mit
HER2-positivem metastasiertem oder lokal rezidivierendem inoperablem Mammakarzinom eine statistisch signifikante Verlängerung der medianen progressionsfreien Überlebenszeit (PFS) um 6,1 Monate, von 12,4 Monate im Kontrollarm auf 18,5 Monate im PertuzumabArm. Dies entspricht einer Reduktion des relativen Risikos um 38\%. Auch die mediane Gesamtüberlebenszeit verlängerte sich signifikant: Nach einer medianen Nachbeobachtungszeit von 30 Monaten zeigte sich eine Reduktion des Sterberisikos um 34\%. Die mediane Gesamtüberlebenszeit war zu diesem Zeitpunkt im Pertuzumab-Arm noch nicht erreicht, im Kontrollarm betrug sie 37,6 Monate.

Die Behandlung mit Pertuzumab induzierte keine zusätzlichen klinisch relevanten Toxizitäten, die Rate kardialer Nebenwirkungen war nicht erhöht. Im Vordergrund der Behandlung mit Chemotherapie plus Antikörper standen Diarrhö, Alopezie, Fatigue, Hautverände-
Der Galenus-von-PergamonPreis würdigt in zwei Kategorien herausragende Arzneimittel-Innovationen und exzellente Forschungsleistung in der klinischen oder experimentellen Pharmakologie.

- Kategorie A wird für eine hervorragende, in Deutschland bereits zugelassene Arzneimittel-Innovation vergeben.

- Kategorie B wird für eine hervorragende Forschungsleistung in der klinischen oder experimentellen Pharmakologie in Deutschland vergeben.

Der Galenus-von-Pergamon-Preis 2013 wird am 17. Oktober 2013 in Berlin verliehen. In diesem Jahr bewerben sich u. a. Takeda mit Adcetris $^{\oplus}$ und Roche mit Perjeta ${ }^{\oplus}$ für den Preis.

rungen, periphere Neuropathien und Neutropenien, die mehrheitlich Grad 1 bis 2 erreichten. Nach Abschluss der Chemotherapie ging die Nebenwirkungsrate deutlich zurück und war in beiden Studienarmen niedrig. Im Langzeitverlauf zeigte sich keine Kumulation der Nebenwirkungen. 